\title{
Molecularly imprinted polymer as micro-solid phase extraction combined with high performance liquid chromatography to determine phenolic compounds in environmental water samples
}

\author{
Qinzhong Feng ${ }^{\mathrm{a}}$, Lixia Zhao ${ }^{\mathrm{a}}$, Jin-Ming Lin ${ }^{\mathrm{b}, *}$ \\ a State Key Laboratory of Environmental Chemistry and Ecotoxicology, Research Center for Eco-Environmental Sciences, Chinese Academy of Sciences, P.O. Box 2871, \\ Beijing 100085, China \\ ${ }^{\mathrm{b}}$ The Key Laboratory of Bioorganic Phosphorus Chemistry \& Chemical Biology, Department of Chemistry, Tsinghua University, Beijing 100084, China
}

\section{A R T I C L E I N F O}

\section{Article history:}

Received 2 February 2009

Received in revised form 26 March 2009

Accepted 13 April 2009

Available online 18 April 2009

\section{Keywords:}

Molecularly imprinted polymer

Micro-solid phase extraction

Precipitation polymerization

2,4,6-Trichlorophenol

Phenolic compounds

High performance liquid chromatography

\begin{abstract}
A B S T R A C T
The molecularly imprinted polymer with 2,4,6-trichlorophenol (2,4,6-TCP) as the template molecule and methylacrylic acid (MAA), divinylbenzene (DVB) as functional monomer and the crosslinker, respectively, has been prepared and used as molecularly imprinted micro-solid phase extraction (MIMSPE) procedure for the selectively preconcentration of phenolic compounds from environmental water samples. Various parameters for the extraction efficiency of the MIMSPE have been evaluated. The optimized MIMSPE method allowed the extraction of the analytes from the sample matrix followed by a selective washing step using acetonitrile containing $0.3 \%(\mathrm{v} / \mathrm{v})$ acetic acid. The characteristics of the MIMSPE method were valid by high performance liquid chromatography. The recoveries ranged between $88.9 \%$ and $102.5 \%$ for tap water, between $80.0 \%$ and $94.0 \%$ for river water, between $80.0 \%$ and $90.5 \%$ for sewage water fortified with $0.4 \mathrm{mg} \mathrm{L}^{-1}$ of phenol, 4-chlorophenol (4-CP), 2,4dichlorophenol (2,4-DCP), 2,4,6-TCP, pentachlorophenol (PCP) were obtained. This method has been successfully applied to preconcentrate and determine of phenolic compounds in environmental water samples directly.
\end{abstract}

(c) 2009 Elsevier B.V. All rights reserved.

\section{Introduction}

Sample pretreatment for the determination of trace amount of phenolic pollutants in environmental water is frequently required in order to attain analyte enrichment and matrix removal. Selective separation technologies, such as liquid chromatography (LC) or gas chromatography (GC) are often used for the quantitative of trace pollutants in environmental samples [1]. However, they are difficult to achieve separation of target compounds from complex matrices directly without the sample pretreatment. In the research into new separation techniques, molecular imprinting technique has gained interest through which we can prepare the sorbent with attractive properties [2].

Technique of molecular imprinting creates special molecular recognition sites in synthetic polymers through the use of template molecules [3-5]. Non-covalent bonding, ionic interactions and hydrophobic interactions are common utilized in the synthesis of molecularly imprinted polymer (MIP) [6-8]. To date, the most widely used method for the preparation of such materials

\footnotetext{
* Corresponding author. Tel.: +8610 62792343; fax: +861062792343.

E-mail address: jmlin@mail.tsinghua.edu.cn (J.-M. Lin).
}

gives particles of irregular shape by bulk method through a low yield, time-consuming crush-and-sieve process [9]. These problems have been solved by using bead polymerization techniques [10], either in two-phase systems by suspension polymerization $[11,12]$, or by grafting techniques [13]. However, these techniques suffer from the presence of water or highly polar organic solvents during the polymerization procedure, which frequently decreases specific interactions between functional monomers and template molecules in most commonly applied non-covalent imprinting mixtures [11]. Hence, for the synthesis of molecularly imprinted micro and nanospheres with monodispersity while retaining high selectivity, a synthetic route avoiding the use of dispersants such as water or polar solvents is demanded. Precipitation techniques have been applied to prepare of imprinted spheres for a variety of different analytes and applications $[14,15]$ because this manipulative procedure is easy to carry out, and there is no need to add stabilizers to the reaction system. Most obtained spherical particles have diameters ranging from microspheres to submicrospheres. The microspheres are ideal for binding assays, capillary electrophoresis, HPLC and solid phase extraction (SPE), but the submicrospheres are not suitable for SPE because the resulting small particles can not be hold back by the frit of the SPE cartridge. Hence, new method for sample pretreatment using submicrosphere 
molecularly imprinted polymer as the sorbent is of great importance.

Micro-solid phase extraction is a novel and feasible alternative to multistep SPE method for preconcentration the analytes in complex samples. In 2006, Basheer et al. [16] reported this approach in which multiwalled carbon nanotube (MWCNTs) was used to extract organophosphorus pesticides (OPPs) from environmental samples. MWCNTs were packed within a porous polypropylene membrane sheet and used for OPP extraction from sewage sludge samples. Like SPE, this extraction procedure involved analyte adsorption followed by solvent desorption but on a much smaller scale. $\mathrm{C}_{18}$ material [17] had also been used as the sorbent to be held within this membrane envelope made of polypropylene to selectively adsorb ketoprofen and ibuprofen in wastewater ranged from 0.03 to $0.08 \mu \mathrm{g} \mathrm{L}^{-1}$. This micro-solid phase extraction technique could combine with other method such as microwave-assisted digestion (MAD) to determine persistent organic pollutants (POPs) in tissue samples with detection limits for POPs ranged from 0.002 to $0.009 \mathrm{ngg}^{-1}$ [18]. Polypropylene membrane envelope is the porous structure with the pore size of $0.22 \mu \mathrm{m}$ which can keep the sorbent in the sheet. The extraction device is easy to prepare in-house at reasonable cost. This technique is robust and durable and a single device can be used for 30 extractions [16]. However, to our best knowledge, there is no yet a report on the use of molecularly imprinted polymers as micro-solid phase extraction sorbent for the separation analytes from complex matrices.

In this article, for the first time, polypropylene membrane sheet is used as the envelope to hold the submicrosphere molecularly imprinted polymer to extract the phenolic compounds from water samples. This molecularly imprinted micro-solid phase extraction (MIMSPE) presents the high specificity, selectivity and sensitivity of the molecular recognition mechanism and the high resolving power of separation methods. It is the method for using submicrosphere molecularly imprinted polymer as the sorbent, furthermore, it is easy to prepare in-house at reasonable cost.

Phenols, particularly chlorinated phenols, are toxic at concentrations in the low $\mu \mathrm{gL}^{-1}$ range and also persistent in the environment. As a consequence, phenolic compounds are listed in the US Environmental Protection Agency (EPA) priority list of pollutants and in Directive 76/464/EEC of the European Union, related to dangerous substances discharged into the aquatic environment, and therefore require regular monitoring [19]. Gao et al. [20] reported that in surface water of China, the concentrations for 2,4-dichlorophenol ranged from $<1.1$ to $19,960.0 \mathrm{ng} \mathrm{L}^{-1}$, with a median level of $5.0 \mathrm{ng} \mathrm{L}^{-1}$. The range of 2,4,6-trichlorophenol was from $<1.4$ to $28,650.0 \mathrm{ng} \mathrm{L}^{-1}$, with a median value of $2.0 \mathrm{ng} \mathrm{L}^{-1}$; the pentachlorophenol concentration ranged from $<1.1$ to $594.0 \mathrm{ng} \mathrm{L}^{-1}$, with a median value of $50.0 \mathrm{ng} \mathrm{L}^{-1}$. Thus, it is necessary to design efficient and sensitive analytical methods which are able to detect trace-level of phenolic compounds. Recently, several papers have been published describing the application of molecular imprinted polymers of halogenated phenols as solid phase extraction materials, and a certain degree of selectivity has been shown [21-24].

In this work, submicrosphere molecularly imprinted polymers were prepared through precipitation polymerization method using 2,4,6-TCP as template molecule, MAA as the functional monomer and DVB as the cross linking agent, toluene and acetonitrile $(1: 3$, $\mathrm{v} / \mathrm{v}$ ) as the porogen. A novel extraction technique, MIMSPE, involving the use of submicrosphere molecularly imprinted polymer wrapped in a porous membrane sheet, was used for selective preconcentration of phenolic compounds and the concentrated phenolic compounds were analyzed with HPLC. The method was validated and successfully used to determine phenolic compounds from environmental water samples.

\section{Experimental}

\subsection{Reagents and apparatus}

The chemicals used for polymer synthesis (2,4,6-TCP, methyacrylic acid (MAA), divinylbenzene (DVB)), and phenol, 4-chlorophenol (4-CP), 2,4-dichlorophenol (2,4-DCP), pentachlorophenol (PCP) were from Acros Organics (Geel, Belgium). 2,2'-Azobisisobutyronitrile (AIBN) was from Shanghai No. 4 Reagent Factory and H.V. Chemical Company (Shanghai, China). Acetonitrile was from J.T. Baker (Phillipsburg, USA). The monomers were purified to remove stabilizers. The AIBN was recrystallized from ethanol. Acetic acid was purchased from Beijing Chemical Reagents Company (Beijing, China), acetonitrile, ethanol and toluene were all from Beijing Beihua Finachemical Company (Beijing, China). Polypropylene membranes (pore size: $0.15,0.22 \mu \mathrm{m}$ ), nylon membranes (pore size: $0.15 \mu \mathrm{m}$ ) and fibre membranes (pore size: $0.15 \mu \mathrm{m}$ ) were purchased from Shenghemo Company (Beijing, China).

The chemicals for HPLC were at least of HPLC grade. Stock standard solutions of phenol, 4-CP, 2,4-DCP, 2,4,6-TCP, PCP were fresh prepared in acetonitrile, and stored at $4{ }^{\circ} \mathrm{C}$ in a refrigerator. All working solutions were prepared by diluting the stock standard solutions with deionized ultrapure water $\left(18.3 \mathrm{M} \Omega \mathrm{cm}^{-1}\right.$, EasyPure LF Barnstead, USA) to the needed concentrations correspondingly.

The resulting precipitation submicroshpere imprinted polymers were characterized by scanning electron microscope (SEM) (S-3000N, Hitachi, Japan) and by nitrogen adsorption/desorption analysis of Brunauer-Emmett-Teller (ASAP 2000, Micromeritics, USA).

To evaluate the polymers, these particles were suspended in water, the solutions were determined with UV-2401PC spectrophotometer (Shimadzu Corporation, Japan).

\subsection{Preparation of molecular imprinted polymers with precipitation methods}

For the preparation of the imprinted polymer, the template molecule 2,4,6-TCP (1 mmol) was dissolved in toluene and acetonitrile $(1: 3, \mathrm{v} / \mathrm{v})(70 \mathrm{~mL})$ solution in a $250-\mathrm{mL}$ thick-walled glass flask, the functional monomer MAA $(8 \mathrm{mmol})$, the cross linking agent DVB $(40 \mathrm{mmol})$ and the initiator AIBN $(0.66 \mathrm{mmol})$ were then added to the solution. The solution was cooled on an ice bath and purged with oxygen-free nitrogen for $10 \mathrm{~min}$. The glass tube was then sealed under nitrogen and placed in a water bath at $60^{\circ} \mathrm{C}$. The reaction was allowed to proceed for $24 \mathrm{~h}$. A reference polymer (NIP) without 2,4,6-TCP was prepared by the same procedure. The polymer particles were washed with methanol-acetic acid $(90: 10, v / v)$ by Soxhlet extraction. Finally, the particles were dried in a vacuum at $35^{\circ} \mathrm{C}$.

\subsection{Preparation of the MIMSPE}

The MIMSPE device consists of the MIPs enclosed within a polypropylene flat-sheet membrane envelope $[16,17]$. To prepare the latter, the longer edge of a rectangular polypropylene membrane was folded over a width of ca. $1.5 \mathrm{~cm}$ and the fold-over section was then trimmed off from the main membrane sheet and cut (at ca. $2-\mathrm{cm}$ intervals) into individual rectangular pieces $(2 \mathrm{~cm} \times 1.5 \mathrm{~cm})$. Two of the three open ends of each piece were then heat-sealed. MIPs were introduced into the device via the remaining open end, which was then heat-sealed to secure the contents. Each device was packed with $15 \mathrm{mg}$ of MIPs. 


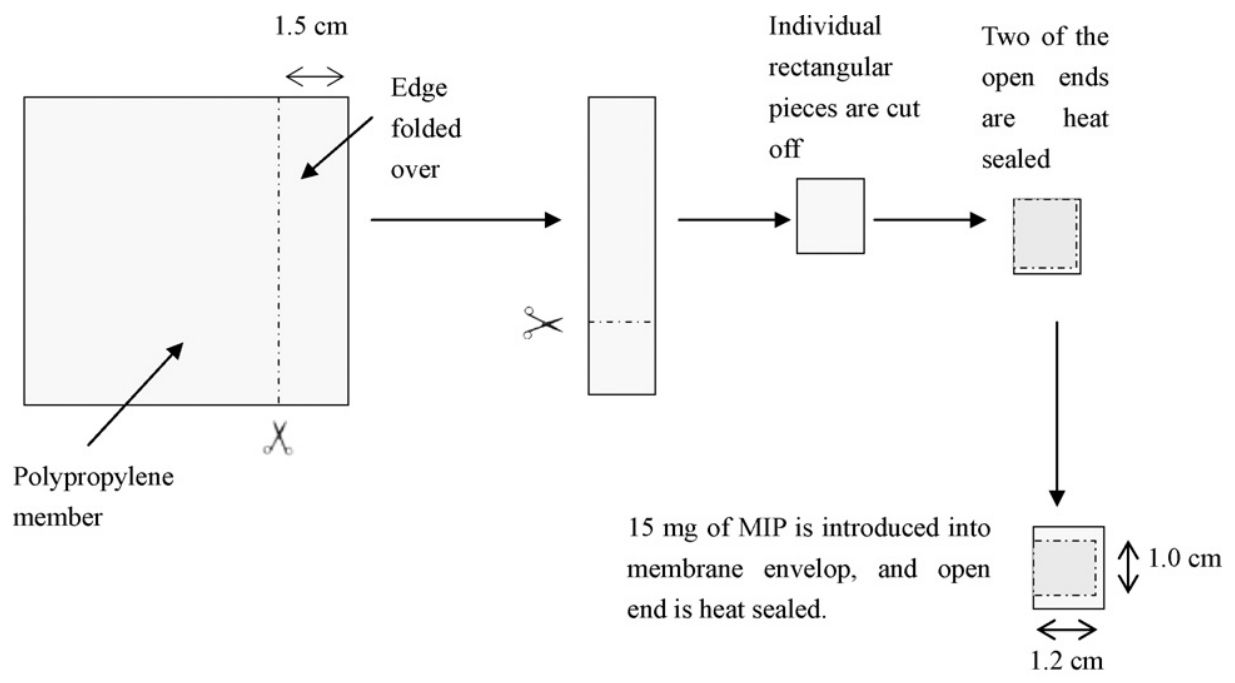

MISPE device

Fig. 1. Schematic of preparation of a MIMSPE device.

The schematic of a typical MIMSPE device is shown in Fig. 1. Each MIMSPE device was conditioned by ultrasonication in ultrapure water and acetonitrile for $2 \mathrm{~min}$. For extraction, the MIMSPE device was placed in aqueous sample and shaken for $50 \mathrm{~min}$. The device tumbled freely within the sample solution during extraction. After extraction, the device was removed, rinsed in ultrapure water, dried with filter paper, and placed in a sample vial. Tweezers were used to handle the device. The analytes were desorbed by ultrasonication in the solution of acetonitrile/acetic acid (99.7:0.3, v/v), out of which $20 \mu \mathrm{L}$ was used for HPLC analysis. The MIMSPE device could be reused after careful rinsing with acetonitrile.

\subsection{Binding characteristic assays}

The binding properties of the MIP to 2,4,6-TCP were studied by batch method as already reported [25]. In a typical rebinding experiment, $5.0 \mathrm{mg}$ washed and dried MIP or NIP were added into $5 \mathrm{~mL}$ solution of 2,4,6-TCP at different concentrations in pure water. The suspensions were then sealed and shaken for $20 \mathrm{~h}$ at room temperature to ensure equilibration. After centrifugation at $3500 \mathrm{rpm}$ for $10 \mathrm{~min}$, the concentration of free 2,4,6-TCP in the supernatant was detected by measuring the absorbance with UV-2401PC spectrophotometer. The amount of 2,4,6-TCP bound to the polymer was calculated by subtracting the concentration of free 2,4,6-TCP from the initial concentration.

\subsection{Determination of 2,4,6-TCP with HPLC}

High performance liquid chromatograph (HPLC, Shimadzu, Japan) for determining the phenolic compounds (phenol, 4-CP, 2,4-DCP, 2,4,6-TCP and PCP) consisted of a binary LC-10ADvp pump, an DGU-12A on-line degasser, a CTO-10Avp column oven, a SPD-10Avp UV-vis detector and SCL-10Avp system controller. The injection loop volume was $20 \mu \mathrm{L}$, and the analytical column was a $25 \mathrm{~cm} \times 0.46 \mathrm{~cm}$ i.d. spherical ODS. The mobile phase consisted of Milli-Q-quality water as solvent A and acetonitrile (containing $0.3 \%(\mathrm{v} / \mathrm{v})$ acetic acid) as solvent B. Both solvent A and solvent $B$ were mixed prior to reaching the analytical column. The flow rate of the mobile phase was $0.5 \mathrm{~mL} \mathrm{~min}^{-1}$, and the gradient profile was $20-40 \%$ B from 0 to $5 \mathrm{~min}, 60 \% \mathrm{~B}$ at $7.5 \mathrm{~min}, 80 \% \mathrm{~B}$ at $20 \mathrm{~min}$, and then isocratic elution for $10 \mathrm{~min}$. The oven temperature was set at $40^{\circ} \mathrm{C}$ then and compounds were detected at
$294 \mathrm{~nm}$, except for phenol and 2,4-DCP which were detected at $280 \mathrm{~nm}$.

\subsection{Sample preparation}

Water samples were collected in glass bottles. After the essential filtration treatment, the collected samples were stored in dark at $4{ }^{\circ} \mathrm{C}$ and the further MIMSPE procedure was accomplished within $24 \mathrm{~h}$ to avoid any microbial degradation of analytes.

Tap water samples were directly collected from the water faucet in our laboratory. River water samples were obtained from the Qinghe River in the northwest of Beijing. The raw sewage samples were collected from influent of sewage treatment plant (STP) which mainly receives domestic water from the uptown (population of $\sim 8000$ ) located around the laboratory.

Filtration is an essential step for real water samples to avoid matrix interference, even for the clean water such as tap water. In this study, freshly collected river water sample and tap water sample were filtered with $0.45 \mu \mathrm{m}$ filter while the sewage water was filtered with $0.20 \mu \mathrm{m}$ filter to remove suspended particles prior to extraction.

\section{Results and discussion}

\subsection{Polymer synthesis and characterization}

Precipitation polymerization is an attractive, simple, and seemingly general method for producing high-quality imprinted products and it is a surfactant-free method that involves polymerization of monomers in dilute solution $(<5 \% \mathrm{w} / \mathrm{v})$ in near- $\theta$ solvents $[15,26]$. Application of precipitation polymerization to molecular imprinting has afforded high-quality, imprinted particles with diameters typically less than $1 \mu \mathrm{m}$ with while the yield of precipitation polymerization method was approximately $85 \%$ [27], which has been applied in analytical techniques such as competition assay [28] and capillary electrochromatography (CEC) [14]. Under optimum polymerization conditions (see Section 2), 2,4,6-TCP molecularly imprinted polymers were prepared. These experiments were performed in triplicate. The particles showed in Fig. 2A are submicrosphere with the average particle diameters of 200-300 nm. BET analysis demonstrated that the polymers were porous and had high specific surface areas which were shown in Table 1. This also showed that NIP and MIP particles were not 
(A)

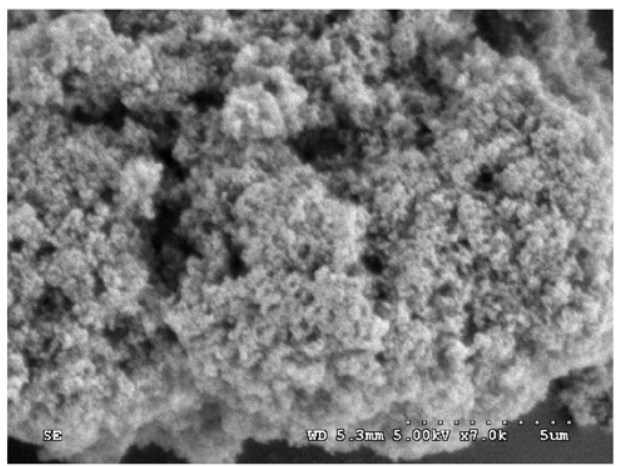

(B)
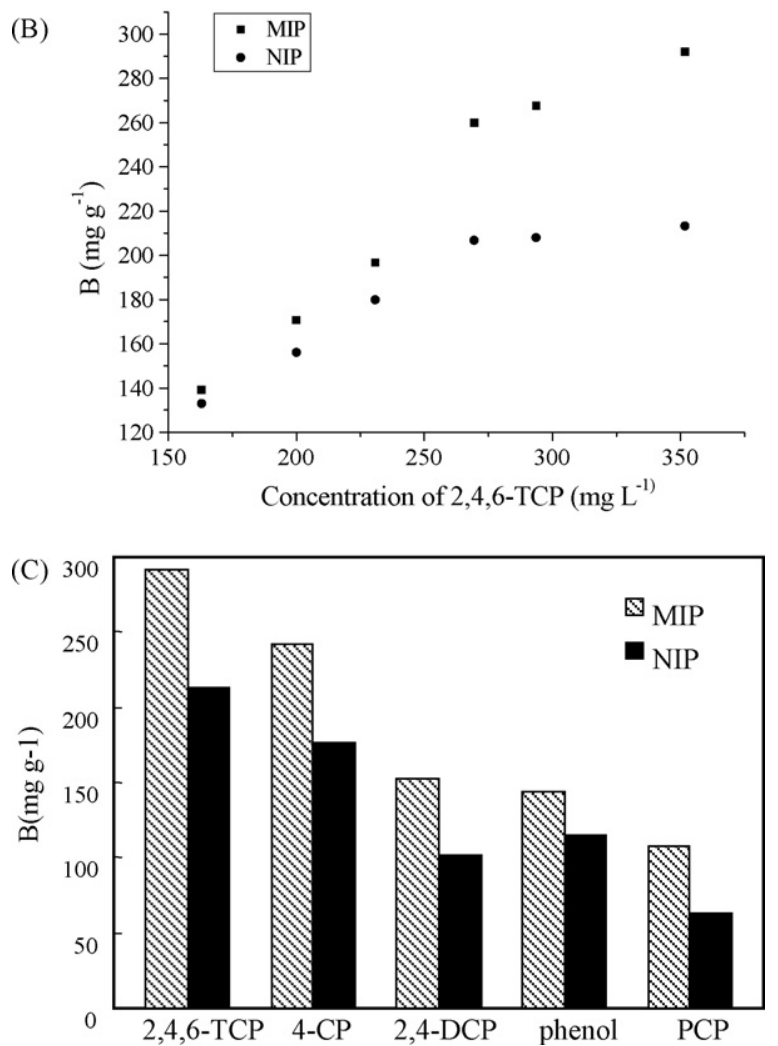

Fig. 2. Characteristic of the imprinted polymer synthesized by precipitation polymerization. ((A) Scanning electron micrographs (SEM) of imprinted polymer; (B) adsorption equilibrium isotherms of 2,4,6-TCP on the MIP and NIP at room temperature; (C) binding selectivity test of 2,4,6-TCP and the structurally related compounds on MIP and NIP).

significantly different in these aspects. Therefore, any differential 2,4,6-TCP loadings displayed by the polymer was not caused by the morphological distinction but by the imprinting effect.

4-Vinylpyridine (4-VP) is particularly well suited for the imprinting templates and provides materials with selectivity of the same order as those obtained using MAA. Theoretically, 2,4,6-TCP is an acid compound which may easily interact with the basic 4-VP, but the experiments showed the reversed results, maybe it is because MAA has the carboxylic acid group serving well as a hydrogen

Table 1

Porosities of polymers determined by BET analysis.

\begin{tabular}{lrr}
\hline Sample & \multicolumn{1}{c}{ MIP } & \multicolumn{1}{c}{ NIP } \\
\hline Pore volume $\left(\mathrm{cm}^{3} \mathrm{~g}^{-1}\right)$ & 1.47 & 1.19 \\
BET surface area $\left(\mathrm{m}^{2} \mathrm{~g}^{-1}\right)$ & 455.94 & 401.21 \\
Pore size $(\AA)$ & 59.71 & 53.57 \\
\hline
\end{tabular}

bond and proton donor as well as a hydrogen bond acceptor in this study, which was agreement with the report that many $\beta$-estradiolimprinted polymers have been prepared using MAA as functional monomer.

\subsection{Evaluated the recognition efficiency of the precipitation imprinted polymer}

The binding assays were carried out as described in Section 2.4 and the experiment was done in water solution instead of acetonitrile since the determination of 2,4,6-TCP was applied in water samples. The experiment was performed in triplicate. The data obtained from batch-type method with 2,4,6-TCP concentration from 10 to $350 \mathrm{mg} \mathrm{L}^{-1}$ were plotted according to the binding isotherm and shown in Fig. 2B. The maximum amount $Q_{\max }$ was estimated to be $292.01 \mathrm{mg} \mathrm{g}^{-1}$ for MIP and $213.32 \mathrm{mg} \mathrm{g}^{-1}$ for NIP. The static adsorption capacity of the 2,4,6-TCP imprinted polymers was more than that of non-imprinted polymers. The results showed that the 2,4,6-TCP imprinted polymers had a higher adsorption capacity for 2,4,6-TCP. They would be more suitable to enrich trace 2,4,6-TCP in the samples. Fig. $2 \mathrm{C}$ showed the selective property of MIP and NIP prepared in this study. The MIPs exhibited high binding affinity for 2,4,6-TCP, as for the other compounds, they also showed certain binding capacity. The imprinting factors that were close to 1.5 for 2,4,6-TCP, 2,4-DCP and PCP and only 1.2 for 4-CP and phenol. It also demonstrated that MIPs could selectively adsorbed these phenolic compounds from water samples.

\subsection{Optimization of the MIMSPE procedure}

A preconcentration process was necessary due to the relative low amount of the analytes in the environmental water. The polymers in the MIMSPE were initially washed with solvent (acetonitrile containing $10 \%(\mathrm{v} / \mathrm{v})$ acetic acid) to remove residual template that could potentially interfere with the analysis, until 2,4,6-TCP was no longer detectable in the eluate. The procedure was carried out as described in Section 2.3, which was determined by UV-2401PC spectrophotometer. The objective of the optimization procedure was to obtain maximum analyte recovery. The parameters investigated were the envelope membrane type, number of MIMSPE devices used for each extraction, the composition and volume of the eluting solvent, extraction time, desorption solvent, desorption time and sample volume. Optimization experiments were performed in triplicate.

\subsubsection{Different kinds of MIMSPE device}

Polypropylene membranes (pore size: $0.15,0.22 \mu \mathrm{m}$ ), nylon membranes (pore size: $0.15 \mu \mathrm{m}$ ), fibre membranes (pore size: $0.15 \mu \mathrm{m}$ ) were evaluated as envelope materials. For the same sorbent, the polypropylene membrane $(0.22 \mu \mathrm{m})$ gave highest analyte enrichment which was about $90 \%$ (Fig. 3A). This may be due to better compatibility of polypropylene with organic solvents or greater matrix interference protection afforded by this material. Fibre membrane was too crisp, it was unsuitable for preparing the MIMSPE devices. Therefore, polypropylene membranes (pore size: $0.22 \mu \mathrm{m}$ ) was used as the envelope materials.

To confirm that polypropylene membranes had little adsorption for 2,4,6-TCP, MIMSPE device with non-MIP was used to absorb 2,4,6-TCP and the results had been compared to that of MIMSPE device with MIP. It was found that the amount of adsorption for $2,4,6$-TCP with MIMSPE device with non-MIP was only $1.0 \%$ of that of MIMSPE device with MIP. Therefore, in the later experiment, the adsorption by the membrane envelope could be ignored. 

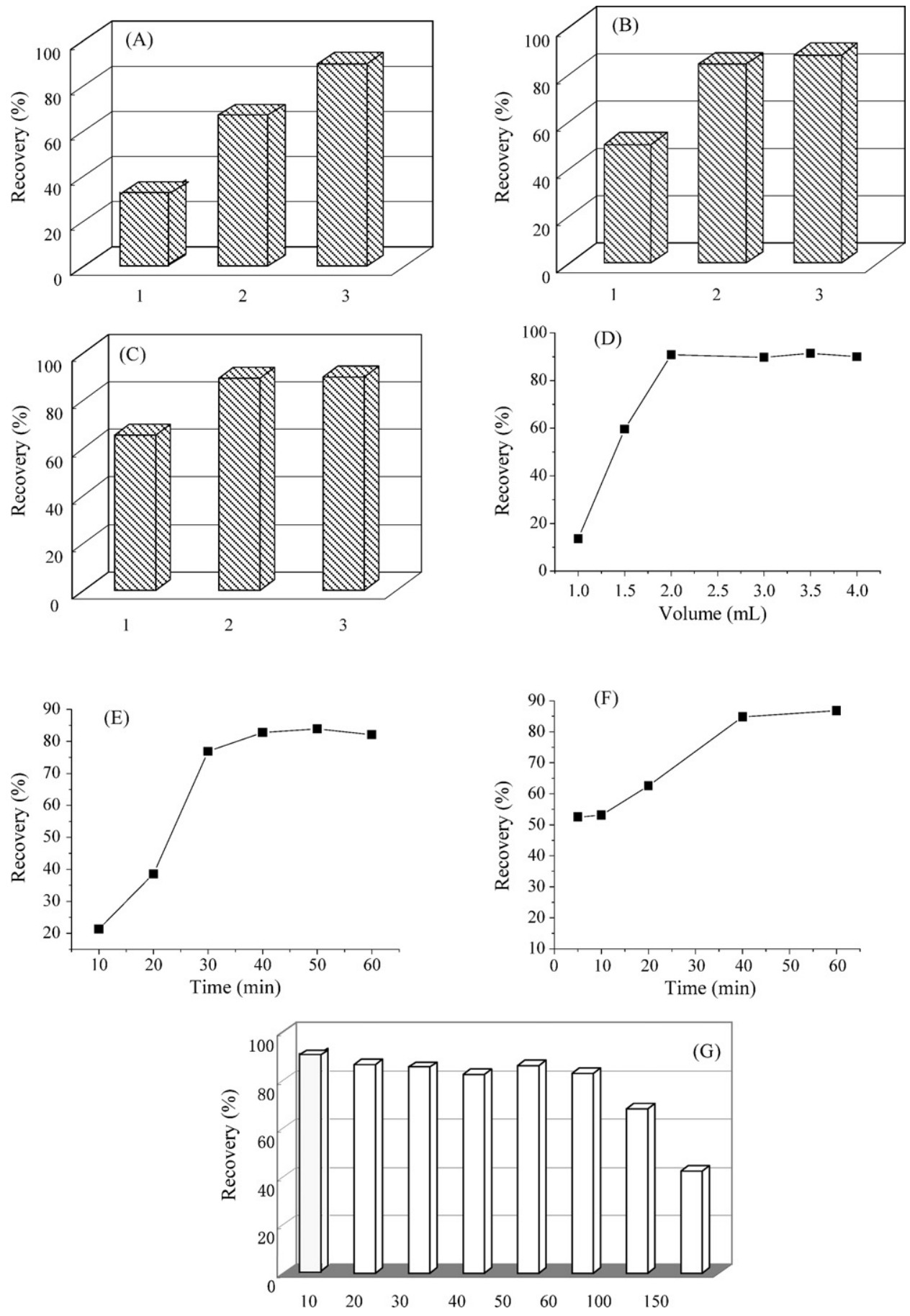

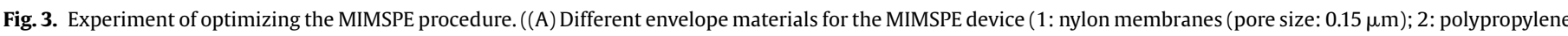

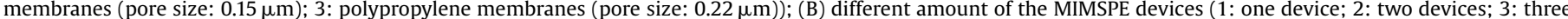

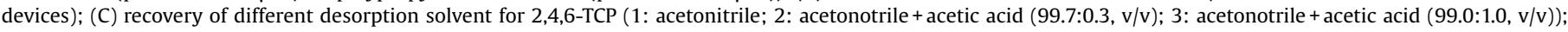

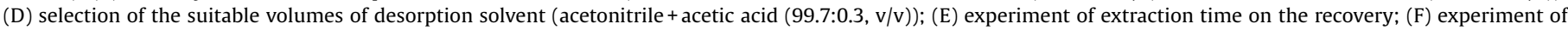
desorption time on the recovery; $(G)$ experiment of sample volume on the recovery).

\subsubsection{Different amount of the MIMSPE devices}

The extraction efficiency based on the use of multiple MIMSPE devices (1-3) was evaluated. As expected, as the number of MIMSPE devices increasing, higher extraction efficiency was observed which was from $50.54 \%$ to $88.63 \%$ (see Fig. 3B), more than two devices could not improve the recovery notably. As the concentration of the analytes in the sample solution considered, two MIMSPE devices exhibited optimum extraction recovery, and additional devices might provide an overcapacity that was not necessary. Furthermore, for less concentrated samples, more than two MIMSPE devices may be useful to increase extraction which should be valued in later experiment.

\subsubsection{Selection of the suitable desorption solvent}

Two MIMSPE devices were added into samples solution containing $0.4 \mathrm{mg} \mathrm{L}^{-1} 2,4,6-\mathrm{TCP}$, and then eluted with $2 \mathrm{~mL}$ of acetonitrile, solvent A (acetonitrile containing $0.3 \%(v / v)$ acetic acid), solvent 
B (acetonitrile containing $1 \%(\mathrm{v} / \mathrm{v})$ acetic acid), respectively. The concentration of the 2,4,6-TCP was determined with HPLC.

To confirm that the acetic acid in the elution solvent (solvent $\mathrm{A}$ and $\mathrm{B}$ ) played an important role in desorbing 2,4,6-TCP from the MIP, pure acetonitrile was tested as the elution solvent. Under these conditions, the $2,4,6$-TCP recovery decreased from $89.9 \%$ to $65.4 \%$. Thus, the addition of acetic acid resulted in an increase in the recovery of 2,4,6-TCP. The most likely explanation was that acetic acid competed with 2,4,6-TCP for the functional groups in the binding sites, but too many acetic acid did not benefit the eluting. From Fig. 3C, we could come to the conclusion that solvent A (acetonitrile containing $0.3 \%(\mathrm{v} / \mathrm{v})$ acetic acid) was the optimum solvent for the following study.

To determine the optimum washing volume, the water sample spiked with $0.4 \mathrm{mg}$ of 2,4,6-TCP was concentrated with the MIP, and different volumes $(1.0,1.5,2.0,3.0,3.5,4.0 \mathrm{~mL})$ of a mixture of solvent $A$ (acetonitrile containing $0.3 \%(\mathrm{v} / \mathrm{v})$ acetic acid) were applied in the washing step, and the eluates were analyzed by HPLC. The results are shown in Fig. 3D, the use of $1.0 \mathrm{~mL}$ of washing solvent allowed recoveries lower than $13.6 \%$ in the MIMSPE device. However, the use of $2.0 \mathrm{~mL}$ of washing solvent allowed recoveries up to $90.8 \%$, which was very acceptable for MIMSPE. In conclusion, a volume of $2 \mathrm{~mL}$ of solvent A (acetonitrile containing $1 \%(\mathrm{v} / \mathrm{v}$ ) acetic acid) was selected for the washing step.

\subsubsection{Effect of extraction time on recovery of the MIMSPE device}

Similar to solid phase microextraction, the MIMSPE procedure is an equilibrium procedure rather than exhaustive extraction procedure. The extraction efficiency of the MIMSPE device depends on partitioning of analyte to sorbent. Extraction duration from 10 to 60 min was investigated to determine equilibrium time (Fig. 3E). Initial partitioning was rapid, followed by a more gradual and protracted uptake; eventually a plateau was reached at about $50 \mathrm{~min}$. 50 min was selected as the optimal extraction time.

\subsubsection{Effect of desorption time on recovery of the MIMSPE device}

Ultrasonication was used to desorb the 2,4,6-TCP from the MIMSPE devices and the desorption time was experimented from 10 to $60 \mathrm{~min}$. Fig. 3F depicted the desorption profile of 2,4,6-TCP showing that 40 -min desorption time was sufficient. No analyte carryover effect was observed with the above conditions.

\subsubsection{Experiment of the sample volume}

The effect of sample volume (from 10 to $150 \mathrm{~mL}, 0.10 \mathrm{mg} \mathrm{L}^{-1}$ ) on extraction efficiency was investigated. Larger extraction efficiencies were observed when sample volumes was increased to $60 \mathrm{~mL}$, this phenomenon was due to increasing analyte enrichment with increasing volume of the sample. A limit to this enrichment was reached when sample volume was $60 \mathrm{~mL}$, it seemed to be the tolerated volume for breakthrough. As shown in Fig. 3G, the extraction efficiency was declined after the sample volume reached at $60 \mathrm{~mL}$. Hence, $60 \mathrm{~mL}$ was selected as the breakthrough volume.

Table 2

The characteristic of the standard curve.

\begin{tabular}{llllll}
\hline $\begin{array}{l}\text { Phenolic } \\
\text { compounds }\end{array}$ & $a$ & $b$ & $R$ & $\begin{array}{l}\text { Linear scope } \\
\left(\mathrm{mg} \mathrm{L}^{-1}\right)\end{array}$ & \multicolumn{1}{l}{$\begin{array}{l}\text { LOD } \\
\left(\mu \mathrm{g} \mathrm{L}^{-1}\right)\end{array}$} \\
\hline Phenol & -0.001 & $5.05 \times 10^{-6}$ & 0.9973 & $0.005-0.5$ & 1.5 \\
4-CP & -0.004 & $2.45 \times 10^{-6}$ & 0.9988 & $0.01-2.0$ & 4.5 \\
$2,4-\mathrm{DCP}$ & 0.0026 & $2.44 \times 10^{-6}$ & 0.9948 & $0.005-0.5$ & 0.56 \\
$2,4,6$-TCP & -0.0123 & $2.48 \times 10^{-6}$ & 0.9982 & $0.005-2.0$ & 1.9 \\
PCP & 0.0072 & $5.26 \times 10^{-6}$ & 0.9966 & $0.01-2.0$ & 4.4 \\
\hline
\end{tabular}

Calibration curves are expressed as regression lines $(y=a+b x)$, where $y$ is integrated peak area and $x$ is concentration of phenolic compounds $\left(\mathrm{mg} \mathrm{L}^{-1}\right)$. $a$ is intercept, $b$ is slope and $R$ is relative coefficient, LOD is limit of detection at 3:1 signal-to-noise ratio.
Table 3

The accuracy and precision in tap water, river water and raw sewage matrixes $(n=3)$.

\begin{tabular}{|c|c|c|c|c|c|c|}
\hline \multirow[t]{2}{*}{ Phenolic compounds } & \multicolumn{2}{|c|}{ Tap water } & \multicolumn{2}{|c|}{ River water } & \multicolumn{2}{|c|}{ Raw sewage } \\
\hline & $R^{\mathrm{a}}(\%)$ & $\operatorname{RSD}^{\mathrm{b}}(\%)$ & $R^{\mathrm{a}}(\%)$ & $\operatorname{RSD}^{\mathrm{b}}(\%)$ & $R^{\mathrm{a}}(\%)$ & $\mathrm{RSD}^{\mathrm{b}}(\%)$ \\
\hline Phenol & 88.9 & 6.6 & 91.7 & 10 & 80.0 & 8.3 \\
\hline 4-CP & 102.5 & 7.8 & 94.0 & 4.2 & 90.5 & 5.3 \\
\hline 2,4-DCP & 92.3 & 0.8 & 83.3 & 3.0 & 82.5 & 6.1 \\
\hline $2,4,6-\mathrm{TCP}$ & 97.1 & 3.9 & 88.3 & 3.8 & 80.0 & 8.8 \\
\hline PCP & 90.0 & 5.6 & 80.0 & 8.8 & 84.4 & 3.2 \\
\hline
\end{tabular}

$R^{\text {a }}$, recovery for the evaluation of accuracy (spiked at $0.10 \mathrm{mg} \mathrm{L}^{-1}$ of each analyte in water samples); $\mathrm{RSD}^{\mathrm{b}}$, relative standard deviation for the evaluation of precision $(n=3)$

\subsection{Determination of phenolic compounds with HPLC}

Determination of phenolic compounds (phenol, 4-CP, 2,4-DCP, 2,4,6-TCP and PCP) with HPLC was carried out as described in Section 2.5. The mobile phase consisted of water, as solvent $A$ and acetonitrile (containing $0.3 \%(\mathrm{v} / \mathrm{v})$ acetic acid) as solvent $B$. The flow rate of the mobile phase was $0.5 \mathrm{~mL} \mathrm{~min}^{-1}$. The oven temperature was set at $40^{\circ} \mathrm{C}$, the injection volume was $20 \mu \mathrm{L}$, and all compounds were detected at $294 \mathrm{~nm}$, except for phenol and 4-CP which were detected at $280 \mathrm{~nm}$. These experiments were performed in triplicate.

The method performance was evaluated by the determination of the linearity, sensitivity, repeatability, and accuracy of the method.

The linearity of the calibration curves were obtained by the determination of the peak areas from analysis of each analyte and the all $r$-values were higher than 0.994 (shown in Table 2).

The limit of detection (LOD), defined as the lowest analyte concentration with a signal-to-noise ratio of 3 , was investigated through the detection of spiked Milli-Q water at serial concentrations. The results showed that the LODs fell between 0.56 and $4.5 \mu \mathrm{g} \mathrm{L}^{-1}$, which indicated this method could be used to detect some polluted water samples.

As shown in Table 3, the recoveries obtained for phenol, 4-CP, 2,4-DCP, 2,4,6-TCP, PCP was in the range of $88.9-102 \%$ for tap water, $80.0-94.0 \%$ for river water and $80.0-90.5 \%$ for sewage water at both fortification levels. The RSD values of targets were lower than $10 \%$ which were also excellent. It demonstrated that 2,4,6-TCP MIPs had a satisfactory performance for the enrichment of phenolic compounds.

\subsection{Analysis of water samples}

The MIMSPE-HPLC method established in this study was applied to quantitatively measure the phenolic compounds in environmental waters. The experiment was performed in triplicate. Table 4 shows that the three compounds could be detected in the Qinghe River, which receives effluent of a large sewage treatment plant (STP). Though the concentrations of 4-CP and 2,4-DCP could not be quantitatively analyzed due to limitation of instrumentation, the concentrations of them were consistent with the usual reports [20](from $<1.1$ to $19,960.0 \mathrm{ng} \mathrm{L}^{-1}$ ) about river which should catch enough concern about their possible environmental risk.

For raw sewage, three compounds were detected. Fig. 4 showed the liquid chromatogram of the phenolic compounds in sewage

Table 4

Concentration of phenolic compounds in river water and raw sewage

\begin{tabular}{lll}
\hline Compound & River water $\left(\mu \mathrm{g} \mathrm{L}^{-1}\right)$ & Raw sewage $\left(\mu \mathrm{g} \mathrm{L}^{-1}\right)$ \\
\hline Phenol & ND & ND \\
$4-$ CP & LOD & 4.7 \\
$2,4-$ DCP & 0.6 & 0.6 \\
$2,4,6-\mathrm{TCP}$ & LOD & 2.0 \\
PCP & ND & ND
\end{tabular}

ND, not detected refers to values below detection limit. 


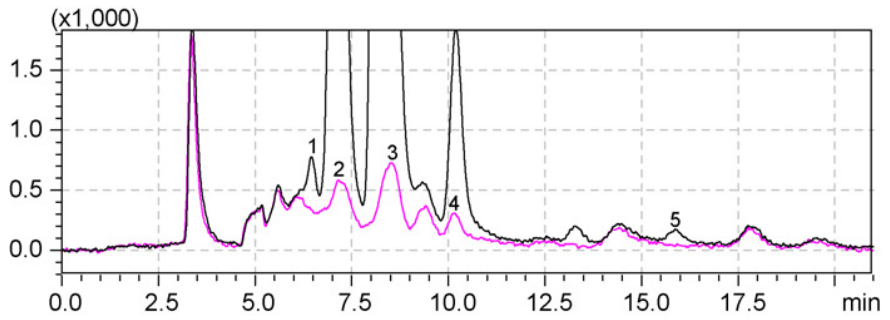

Fig. 4. Chromatograms of sewage water sample before (bottom line) and after (top line) spiked at $0.4 \mathrm{mg} \mathrm{L}^{-1}$ of each analyte. Peak identifications: (1) phenol, (2) 4-CP, (3) 2,4-DCP, (4) 2,4,6-TCP, (5) PCP.

water before and after spiking at $0.4 \mathrm{mg} \mathrm{L}^{-1}$. It indicted that the matrix effects on MIMSPE were negligible. Phenolic compounds can be released into the environment directly and indirectly, such as industrial effluents, conversion products from natural and synthetic chemicals, including pesticides [29], but for the raw sewage in this study, these chlorophenols may be generated from the chlorination treatments in drinking water disinfection [30] or from lignin degradation in the production of paper [31].

\section{Conclusions}

The 2,4,6-TCP imprinted polymer was prepared by precipitation polymerization using MAA and DVB as the monomer and crosslinker, respectively. The obtained polymer showed good selectivity and enrichment efficiency. Thus, a method was successfully developed by using the MAA-Co-DVB polymer as the MIMSPE coupled with HPLC for enrichment and analysis of phenolic compounds in environmental water. The high recoveries (80.0-102.5\%) and satisfied precision $(0.8-10.0 \%)$ for all the analytes proved that the method was valid for the analysis of some phenolic compounds mentioned above in different matrixes (tap water, river water, sewage water). From this point of view, the MIMSPE-HPLC method was developed to determine some phenolic compounds in environmental water directly and the results of the recovery validated the method.

\section{Acknowledgements}

This work was supported by the National Natural Science Foundation of China (Grant No. 20728505) and the Sino-German Center for Research Promotion (DFG and NSFC, GZ 364).

\section{References}

[1] H.M. Oliveira, M.A. Segundo, J.L.F.C. Lima, V. Cerda, Talanta 77 (2009) 1466.

[2] H. Yang, S. Zhang, F. Tan, Z. Zhuang, X. Wang, J. Am. Chem. Soc. 127 (2005) 1378.

[3] C. Lu, W. Zhou, B. Han, H. Yang, X. Chen, X. Wang, Anal. Chem. 79 (2007) 5457.

[4] G. Wulff, Chem. Rev. 102 (2002) 1.

[5] K. Nemoto, T. Kubo, M. Nomachi, T. Sano, T. Matsumoto, K. Hosoya, T. Hattori, K Kaya, J. Am. Chem. Soc. 129 (2007) 13626.

[6] C. Xie, B. Liu, Z. Wang, D. Gao, G. Guan, Z. Zhang, Anal. Chem. 80 (2008) 437.

[7] N. Masque, R.M. Marce, F. Borrull, P.A.G. Cormack, D.C. Sherrington, Anal. Chem 72 (2000) 4122.

[8] J.-M. Lin, M. Yamada, Anal. Chem. 72 (2000) 1148.

[9] J. Oxelbark, C. Legido-Quigley, C.S.A. Aureliano, M.M. Titirici, E. Schillinger, B. Sellergren, J. Courtois, K. Irgum, L. Dambies, P.A.G. Cormack, D.C. Sherrington, E. De Lorenzi, J. Chromatogr. A 1160 (2007) 215.

[10] H. Kempe, M. Kempe, Anal. Chem. 78 (2006) 3659.

[11] A.G. Mayes, K. Mosbach, Anal. Chem. 68 (1996) 3769.

[12] H. Kempe, M. Kempe, Macromol. Rapid Commun. 25 (2004) 315.

[13] O. Prucker, J. Ruhe, Macromolecules 31 (1998) 602.

[14] T. de Boer, R. Mol, R.A. de Zeeuw, G.J. de Jong, D.C. Sherrington, P.A.G. Cormack, K. Ensing, Electrophoresis 23 (2002) 1296.

[15] H. Sambe, K. Hoshina, R. Moaddel, I.W. Wainer, J. Haginaka, J. Chromatogr. A 1134 (2006) 88.

[16] C. Basheer, A.A. Ainedhary, B.S.M. Rao, S. Valliyaveettil, H.K. Lee, Anal. Chem. 78 (2006) 2853.

[17] C. Basheer, H.G. Chong, T.M. Hii, H.K. Lee, Anal. Chem. 79 (2007) 6845.

[18] C. Basheer, K. Narasirnhan, M. Yin, C. Zhao, M. Choolani, H.K. Lee, J. Chromatogr. A 1186 (2008) 358

[19] R. Wissiack, E. Rosenberg, M. Grasserbauer, J. Chromatogr. A 896 (2000) 159.

[20] J. Gao, L. Liu, X. Liu, H. Zhou, S. Huang, Z. Wang, Chemosphere 71 (2008) 1181.

[21] C. Nicholls, K. Karim, S. Piletsky, S. Saini, S. Setford, Biosens. Bioelectron. 21 (2006) 1171.

[22] C. Baggiani, L. Anfossi, C. Giovannoli, C. Tozzi, J. Chromatogr. B 804 (2004) 31.

[23] T. Kubo, K. Hosoya, Y. Watabe, T. Ikegami, N. Tanaka, T. Sano, K. Kaya, J. Chromatogr. A 1029 (2004) 37.

[24] E. Caro, R.M. Marce, P.A.G. Cormack, D.C. Sherrington, F. Borrull, J. Chromatogr. A 995 (2003) 233.

[25] Y. Xiong, H. Zhou, Z. Zhang, D. He, C. He, Spectrochim. Acta A 66 (2007) 341.

[26] W. Li, H.D.H. Stover, Macromolecules 33 (2000) 4354

[27] L. Ye, R. Weiss, K. Mosbach, Macromolecules 33 (2000) 8239-8245.

[28] L. Ye, P.A.G. Cormack, K. Mosbach, Anal. Commun. 36 (1999) 35

[29] H. Bagheri, M. Saraji, J. Chromatogr. A 910 (2001) 87.

[30] M.L. Davi, F. Gnudi, Water Res. 33 (1999) 3213.

[31] I. Rodriguez, M.P. Llompart, R. Cela, J. Chromatogr. A 885 (2000) 291 